Document downloaded from:

http://hdl.handle.net/10251/46730

This paper must be cited as:

Castro Company, F.; Romaguera Bonilla, S.; Tirado Peláez, P. (2011). The bicompletion of fuzzy quasi-metric spaces. Fuzzy Sets and Systems. 166(1):56-64.

doi::10.1016/j.fss.2010.12.004.

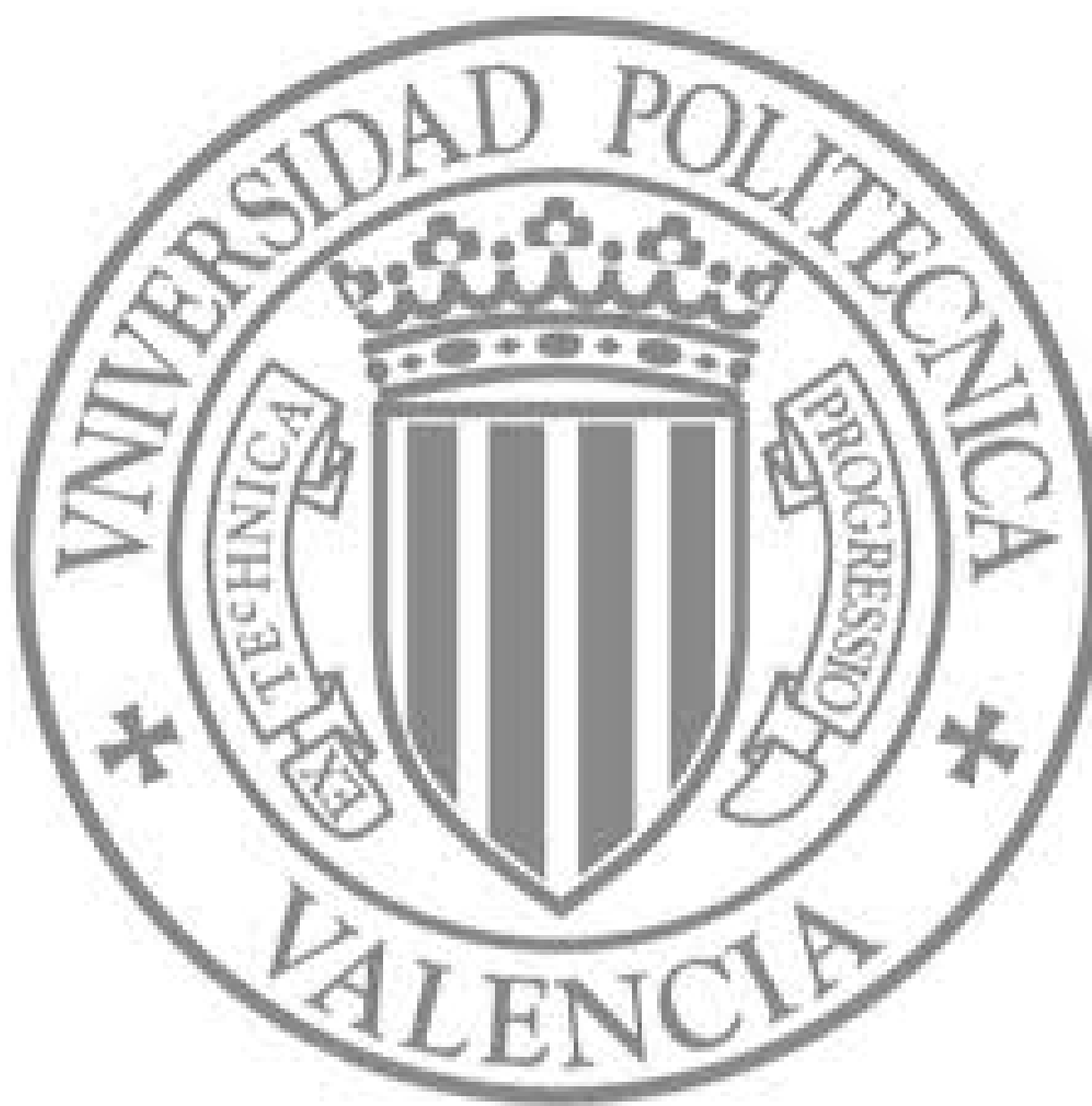

The final publication is available at

http://dx.doi.org/10.1016/j.fss.2010.12.004

Copyright Elsevier 


\title{
The bicompletion of fuzzy quasi-metric spaces
}

\author{
F. Castro-Company ${ }^{\mathrm{a}, 1}$ S. Romaguera ${ }^{\mathrm{b}, *, 1} \mathrm{P}$. Tirado ${ }^{\mathrm{b}, 1}$ \\ ${ }^{a}$ Departamento de Matemática Aplicada, Universidad Politécnica de Valencia, \\ 46071 Valencia, Spain \\ ${ }^{\mathrm{b}}$ Instituto Universitario de Matemática Pura y Aplicada, Universidad Politécnica \\ de Valencia, 46071 Valencia, Spain
}

\begin{abstract}
Extending the well-known result that every fuzzy metric space, in the sense of Kramosil and Michalek, has a completion which is unique up to isometry, we show that every KM-fuzzy quasi-metric space has a bicompletion which is unique up to isometry, and deduce that for each KM-fuzzy quasi-metric space, the completion of its induced fuzzy metric space coincides with the fuzzy metric space induced by its bicompletion.
\end{abstract}

Key words: Fuzzy quasi-metric; Bicomplete; Isometry; Bicompletion.

\section{Introduction}

Kramosil and Michalek introduced in [11] their celebrated notion of a fuzzy metric space and established its relation with the notion of a probabilistic metric space. In particular, they observed that the class of fuzzy metric spaces, in their sense, is "equivalent" to the class of Menger spaces having a continuous t-norm. Sherwood proved in [21] that every Menger space belonging to this class has a completion which is unique up to isometry, and thus one can easily deduce that every fuzzy metric space has a completion which is unique up to isometry.

* Corresponding author.

Email addresses: fracasco@mat.upv.es (F. Castro-Company), sromague@mat.upv.es (S. Romaguera), pedtipe@mat.upv.es (P. Tirado).

1 Supported by the Spanish Ministry of Science and Innovation, under grant MTM2009-12872-C02-01. 
The concept of metric fuzzy metric space was generalized to the quasi-metric setting in [1] and [9], where several properties of these structures were discussed. Recently, it were given in [16], [17] and [18], applications of fixed point theorems, in the realm of fuzzy quasi-metric spaces, to deduce the existence and uniqueness of solution for the recurrence equations associated to some types of algorithms, whereas in [15] it was presented a study of a notion of Hausdorff fuzzy quasi-pseudo-metric on the collection of nonempty subsets of a given fuzzy quasi-metric space.

In this context, the completion of fuzzy quasi-metric spaces appears as a natural and attractive question, which will be discussed here. In fact, we show that every fuzzy quasi-metric space (in the sense of Definition 2 below) has a (fuzzy quasi-metric) bicompletion which is unique up to isometry. Then, the completion of a fuzzy metric space is restated as a particular case, and we deduce that for each fuzzy quasi-metric space, the completion of its induced fuzzy metric space coincides with the fuzzy metric space induced by its bicompletion.Some illustrative example are also given. We emphasize at this point the while Sherwood's construction is strongly based on the properties of Lévy's metric (see [6,14] for its definition and properties), our construction avoids the use of Lévy's metric and directly uses suprema of subsets of $[0,1]$ and lower limits of sequences in $[0,1]$.

\section{Basic notions and preliminary results}

Our basic references for quasi-uniform and quasi-metric spaces are $[3,12]$.

Following the modern terminology, by a quasi-metric on a set $X$ we mean a function $d: X \times X \rightarrow[0, \infty)$ such that for all $x, y, z \in X:$ (i) $x=y \Leftrightarrow$ $d(x, y)=d(y, x)=0$; (ii) $d(x, z) \leq d(x, y)+d(y, z)$.

A quasi-metric space is a pair $(X, d)$ such that $X$ is a set and $d$ is a quasi-metric on $X$.

Each quasi-metric $d$ on $X$ induces a $T_{0}$ topology $\tau_{d}$ on $X$ which has as a base the family of open balls $\left\{B_{d}(x, r): x \in X, \varepsilon>0\right\}$, where $B_{d}(x, \varepsilon)=\{y \in X$ : $d(x, y)<\varepsilon\}$ for all $x \in X$ and $\varepsilon>0$.

Given a quasi-metric $d$ on $X$, then the function $d^{-1}$ defined by $d^{-1}(x, y)=$ $d(y, x)$, is also a quasi-metric on $X$, called the conjugate of $d$, and the function $d^{s}$ defined by $d^{s}(x, y)=\max \left\{d(x, y), d^{-1}(x, y)\right\}$ is a metric on $X$.

A quasi-metric space $(X, d)$ is said to be bicomplete if $\left(X, d^{s}\right)$ is a complete metric space. In this case we say that $d$ is a bicomplete quasi-metric on $X$. 
A topological space $(X, \tau)$ is called quasi-metrizable if there is a quasi-metric $d$ on $X$ such that $\tau=\tau_{d}$.

According to [20], a binary operation $*:[0,1] \times[0,1] \rightarrow[0,1]$ is a continuous t-norm if $*$ satisfies the following conditions: $(\mathrm{i}) *$ is associative and commutative; (ii) $*$ is continuous; (iii) $a * 1=a$ for every $a \in[0,1]$; (iv) $a * b \leq c * d$ whenever $a \leq c$ and $b \leq d$, with $a, b, c, d \in[0,1]$.

Three paradigmatic examples of continuous t-norms are $\wedge$, Prod and $*_{L}$ (the Lukasiewicz t-norm), which are defined by $a \wedge b=\min \{a, b\}, a \operatorname{Prod} b=a \cdot b$ and $a *_{L} b=\max \{a+b-1,0\}$, respectively.

Note that by conditions (iii), (iv), above, $* \leq \wedge$ for every continuous t-norm $*$.

Definition 1 ([1,9]). A KM-fuzzy quasi-metric on a set $X$ is a pair $(M, *)$ such that $*$ is a continuous t-norm and $M$ is a fuzzy set in $X \times X \times[0, \infty)$ such that for all $x, y, z \in X$ :

(KM1) $M(x, y, 0)=0$

(KM2) $x=y$ if and only if $M(x, y, t)=M(y, x, t)=1$ for all $t>0$;

(KM3) $M(x, z, t+s) \geq M(x, y, t) * M(y, z, s)$ for all $t, s \geq 0$

$(\mathrm{KM} 4) M\left(x, y,_{-}\right):[0, \infty) \rightarrow[0,1]$ is left continuous.

A KM-fuzzy quasi-metric $(M, *)$ on $X$ such that for each $x, y \in X$ :

(KM5) $M(x, y, t)=M(y, x, t)$ for all $t>0$

is said to be a fuzzy metric on $X$ (in the sense of Kramosil and Michalek [11]).

In the following, KM-fuzzy quasi-metrics will be simply called fuzzy quasimetrics if no confusion arises.

Definition 2 ([1,9]). A fuzzy (quasi-)metric space is a triple $(X, M, *)$ such that $X$ is a set and $(M, *)$ is a fuzzy (quasi-)metric on $X$.

If $(M, *)$ is a fuzzy quasi-metric on a set $X$, it is obvious that $\left(M^{-1}, *\right)$ is also a fuzzy quasi-metric on $X$, where $M^{-1}$ is the fuzzy set in $X \times X \times[0, \infty)$ defined by $M^{-1}(x, y, t)=M(y, x, t)$. Moreover, if we denote by $M^{i}$ the fuzzy set in $X \times X \times[0, \infty)$ given by $M^{i}(x, y, t)=\min \left\{M(x, y, t), M^{-1}(x, y, t)\right\}$, 
then $\left(M^{i}, *\right)$ is, clearly, a fuzzy metric on $X$. We shall refer to $\left(X, M^{i}, *\right)$ as the fuzzy metric space induced by $(X, M, *)$.

Similarly to the fuzzy metric case, each fuzzy quasi-metric $(M, *)$ on a set $X$ induces a $T_{0}$ topology $\tau_{M}$ on $X$ which has as a base the family of open balls $\left\{B_{M}(x, \varepsilon, t): x \in X, 0<\varepsilon<1, t>0\right\}$, where $B_{M}(x, \varepsilon, t)=\{y \in X:$ $M(x, y, t)>1-\varepsilon\}($ see $[9,10])$.

The following useful facts are well-known $([1,9])$ :

(a) If $(M, *)$ is a fuzzy quasi-metric space on a set $X$, then, for each $x, y \in X$ the function $M\left(x, y,{ }_{-}\right)$is nondecreasing.

(b) A sequence $\left(x_{n}\right)_{n}$ in a fuzzy quasi-metric space $(X, M, *)$ converges to a point $x \in X$ with respect to $\tau_{M}$ if and only if $\lim _{n} M\left(x, x_{n}, t\right)=1$ for all $t>0$.

Example 1. Let $(X, d)$ be a quasi-metric space and let $*$ be a continuous t-norm. Then, as in the metric case, the pair $\left(M_{d, 01}, *\right)$ is a fuzzy quasi-metric on $X$, where $M$ is the fuzzy set in $X \times X \times[0, \infty)$ given by $M_{d, 01}(x, y, t)=0$ if $d(x, y) \geq t$ and $M_{d, 01}(x, y, t)=1$ if $d(x, y)<t$. Moreover, the topology $\tau_{d}$, induced by $d$, coincides with the topology $\tau_{M_{d, 01}}$ induced by $\left(M_{d, 01}, *\right)$.

We say that a topological space $(X, \tau)$ admits a compatible fuzzy quasi-metric if there is a fuzzy quasi-metric $(M, *)$ on $X$ such that $\tau=\tau_{M}$.

Then, it follows from Example 1 above that every quasi-metrizable topological space admits a compatible fuzzy quasi-metric.

Conversely, it was shown in [9] that for each fuzzy quasi-metric space $(X, M, *)$, the countable family $\left\{U_{n}: n=2,3, \ldots\right\}$ is a base for a quasi-uniformity $\mathcal{U}_{M}$ on $X$ compatible with $\tau_{M}$, where $U_{n}=\{(x, y) \in X \times X: M(x, y, 1 / n)>1-1 / n\}$.

Consequently, for every fuzzy quasi-metric space $(X, M, *)$, the topological space $\left(X, \tau_{M}\right)$ is quasi-metrizable.

Moreover, the conjugate quasi-uniformity $\left(\mathcal{U}_{M}\right)^{-1}$ coincides with $\mathcal{U}_{M^{-1}}$ and it is compatible with $\tau_{M^{-1}}$.

The following example shows that in case that the continuous t-norm $*$ is greater or equal to $*_{L}$, it is possible to explicitly construct a compatible quasimetric.

Example 2. Let $(X, M, *)$ be a fuzzy quasi-metric space such that $*_{L} \leq *$. Similarly to the metric case, the function $d_{M}: X \times X \rightarrow[0,1]$ given by 
$d_{M}(x, y)=\sup \{t \geq 0: 1-M(x, y, t) \geq t\}$, is a quasi-metric on $X$ whose induced topology coincides with $\tau_{M}$ (compare [1, Remark 7.6.1]).

Remark 1. If in Definition 1, we assume that $M$ is a fuzzy set in $X \times X \times$ $(0, \infty)$, and conditions (KM2) and (KM4) are replaced, respectively, by:

$(\mathrm{GV} 2) x=y$ if and only if $M(x, y, t)=M(y, x, t)=1$ for some $t>0$; and

$(\mathrm{GV} 4) M\left(x, y,{ }_{-}\right):(0, \infty) \rightarrow(0,1]$ is continuous,

then $(M, *)$ is called a $\mathrm{GV}$-fuzzy quasi-metric on $X$, and $(X, M, *)$ is called a GV-fuzzy quasi-metric space $([9])$. If, in addition, $(M, *)$ satisfies the symmetry condition (KM5), then $(X, M, *)$ is a fuzzy metric space in the sense of George and Veeramani $([4,5])$, and we will refer to it as a GV-fuzzy metric space. Obviously, each GV-fuzzy (quasi-)metric space $(X, M, *)$ can be considered as a fuzzy (quasi-)metric space, in the sense of Definition 1, by defining $M(x, y, 0)=0$ for all $x, y \in X$. Therefore, each GV-fuzzy quasi-metric space induces a topology $\tau_{M}$ defined as in the KM-case. Moreover, the properties of KM-fuzzy quasi-metrics given above remain valid for GV-fuzzy quasi-metrics.

We conclude this section with a paradigmatic example which shows that, actually, each quasi-metric induces a compatible GV-fuzzy quasi-metric (compare Example 1).

Example $3([9])$. Let $(X, d)$ be a quasi-metric space and let $M_{d}$ be the function defined on $X \times X \times(0, \infty)$ by

$$
M_{d}(x, y, t)=\frac{t}{t+d(x, y)}
$$

for all $t>0$. Then, for each continuous t-norm $*,\left(M_{d}, *\right)$ is a GV-fuzzy quasimetric on $X$ called the GV-fuzzy quasi-metric induced by $d$, and $\left(X, M_{d}, *\right)$ is called the standard GV-fuzzy quasi-metric space of $(X, d)$. Furthermore, it is easy to check that $\left(M_{d}\right)^{-1}=M_{d^{-1}}$ and $\left(M_{d}\right)^{i}=M_{d^{s}}$. Finally, the topology $\tau_{d}$ coincides with the topology $\tau_{M_{d}}$.

\section{The bicompletion of a fuzzy quasi-metric space}

Let us recall (compare [5]) that a fuzzy metric space $(X, M, *)$ is complete provided that every Cauchy sequence converges with respect to $\tau_{M}$, where a 
sequence $\left(x_{n}\right)_{n}$ is Cauchy provided that for each $\varepsilon \in(0,1)$ and each $t>0$, there exists an $n_{0}$ such that $M\left(x_{n}, x_{m}, t\right)>1-\varepsilon$ for all $n, m \geq n_{0}$.

According to $[9,10]$, a fuzzy quasi-metric space $(X, M, *)$ is called bicomplete if $\left(X, M^{i}, *\right)$ is a complete fuzzy metric space. In this case, we say that $(M, *)$ is a bicomplete fuzzy quasi-metric on $X$.

Definition $3([9,10])$. A mapping $f$ from a fuzzy quasi-metric space $(X, M, *)$ to a fuzzy quasi-metric space $(Y, N, \star)$ is said to be an isometry if $M(x, y, t)=$ $N(f(x), f(y), t)$ for each $x, y \in X$ and each $t>0$.

The fuzzy quasi-metric spaces $(X, M, *)$ and $(Y, N, \star)$ are called isometric if there is an isometry from $X$ onto $Y$.

Definition $4([9,10])$. Let $(X, M, *)$ be a fuzzy quasi-metric space. A (fuzzy quasi-metric) bicompletion of $(X, M, *)$ is a bicomplete fuzzy quasi-metric space $(Y, N, \star)$ such that $(X, M, *)$ is isometric to a $\tau_{N^{i}}$-dense subspace of $Y$.

In the sequel we shall construct the bicompletion of a fuzzy quasi-metric space.

Indeed, let $(X, M, *)$ be a fuzzy quasi-metric space.

Denote by $S$ the collection of all Cauchy sequences in $\left(X, M^{i}, *\right)$.

Define a relation $\sim$ on $S$ by

$$
\left(x_{n}\right)_{n} \sim\left(y_{n}\right)_{n} \Longleftrightarrow \sup _{0<s<t} \underline{\lim } M^{i}\left(x_{n}, y_{n}, s\right)=1 \quad \text { for all } t>0
$$

where by $\underline{\lim } M^{i}\left(x_{n}, y_{n}, s\right)$ we denote, as usual, the lower limit of the sequence $\left(M^{i}\left(x_{n}, y_{n}, s\right)\right)_{n}$, i.e.,

$$
\underline{\lim } M^{i}\left(x_{n}, y_{n}, s\right)=\sup _{k} \inf _{n \geq k} M^{i}\left(x_{n}, y_{n}, s\right) .
$$

Then we have:

Lemma 1. $\sim$ is an equivalence relation on $S$.

Proof. Let $\left(x_{n}\right)_{n},\left(y_{n}\right)_{n},\left(z_{n}\right)_{n} \in S$. Clearly $\left(x_{n}\right)_{n} \sim\left(x_{n}\right)_{n}$ because $M^{i}\left(x_{n}, x_{n}, s\right)=$ 
1 for all $n \in \mathbb{N}$ and $s>0$, so that for each $t>0$,

$$
\sup _{0<s<t} \underline{\lim } M^{i}\left(x_{n}, x_{n}, s\right)=1 \text {. }
$$

Moreover, if $\left(x_{n}\right)_{n} \sim\left(y_{n}\right)_{n}$, it immediately follows that $\left(y_{n}\right)_{n} \sim\left(x_{n}\right)_{n}$ because $M^{i}\left(x_{n}, y_{n}, s\right)=M^{i}\left(y_{n}, x_{n}, s\right)$ for all $n \in \mathbb{N}$ and $s>0$, so that for each $t>0$,

$$
\sup _{0<s<t} \underline{\lim } M^{i}\left(y_{n}, x_{n}, s\right)=\sup _{0<s<t} \underline{\lim } M^{i}\left(x_{n}, y_{n}, s\right)=1 \text {. }
$$

Finally, suppose that $\left(x_{n}\right)_{n} \sim\left(y_{n}\right)_{n}$ and $\left(y_{n}\right)_{n} \sim\left(z_{n}\right)_{n}$. Let $t>0$. We shall prove that $\sup _{0<s<t} \underline{\lim } M^{i}\left(x_{n}, z_{n}, s\right)=1$.

To this end, choose an arbitrary $\varepsilon \in(0,1)$. Then, there exists $\delta \in(0,1)$ such that $(1-\delta) *(1-\delta)>1-\varepsilon$. Hence, there exists $s^{\prime} \in(0, t)$ such that $\underline{\lim } M^{i}\left(x_{n}, y_{n}, s^{\prime}\right)>1-\delta$, and consequently there exists $k_{1} \in \mathbb{N}$ such that

$$
M^{i}\left(x_{n}, y_{n}, s^{\prime}\right)>1-\delta
$$

for all $n \geq k_{1}$.

Now choose $r>0$ such that $s^{\prime}+r<t$. Since $\left(y_{n}\right)_{n} \sim\left(z_{n}\right)_{n}$, we have that $\sup _{0<s<r} \underline{\lim } M^{i}\left(y_{n}, z_{n}, s\right)=1$. Hence, there exist $s^{\prime \prime} \in(0, r)$ and $k_{2} \geq k_{1}$ such that

$$
M^{i}\left(y_{n}, z_{n}, s^{\prime \prime}\right)>1-\delta
$$

for all $n \geq k_{2}$.

Therefore

$$
M^{i}\left(x_{n}, z_{n}, s^{\prime}+s^{\prime \prime}\right) \geq M^{i}\left(x_{n}, y_{n}, s^{\prime}\right) * M^{i}\left(y_{n}, z_{n}, s^{\prime \prime}\right) \geq(1-\delta) *(1-\delta)>1-\varepsilon,
$$

for all $n \geq k_{2}$, which implies that

$$
\underline{\lim } M^{i}\left(x_{n}, z_{n}, s^{\prime}+s^{\prime \prime}\right) \geq 1-\varepsilon .
$$

Since $0<s^{\prime}+s^{\prime \prime}<t$, we deduce that

$$
\sup _{0<s<t} \underline{\lim } M^{i}\left(x_{n}, z_{n}, t\right)=1,
$$

and hence $\left(x_{n}\right)_{n} \sim\left(z_{n}\right)_{n}$ 
Now define a function $M_{S}: S \times S \times[0, \infty) \rightarrow[0,1]$ as follows:

$$
M_{S}\left(\left(x_{n}\right)_{n},\left(y_{n}\right)_{n}, 0\right)=0
$$

and

$$
M_{S}\left(\left(x_{n}\right)_{n},\left(y_{n}\right)_{n}, t\right)=\sup _{0<s<t} \underline{\lim } M\left(x_{n}, y_{n}, s\right)
$$

for all $t>0$.

Then:

Lemma 2. $M_{S}$ satisfies conditions (KM1), (KM3) and (KM4) of Definition 1. (In fact, $M_{s}$ is a KM-fuzzy quasi-pseudo-metric in the sense of [9]).

Proof. Condition (KM1) is obviously satisfied by the definition of $M_{S}$.

Let $\left(x_{n}\right)_{n},\left(y_{n}\right)_{n},\left(z_{n}\right)_{n} \in S ., t, s>0$, and put $\alpha=M_{S}\left(\left(x_{n}\right)_{n},\left(y_{n}\right)_{n}, t\right), \beta=$ $M_{S}\left(\left(y_{n}\right)_{n},\left(z_{n}\right)_{n}, s\right)$ and $\gamma=M_{S}\left(\left(x_{n}\right)_{n},\left(z_{n}\right)_{n}, t+s\right)$. We shall show that $\alpha * \beta \leq$ $\gamma$

If $\alpha=0$ or $\beta=0$, the conclusion is obvious. So we assume that $\alpha>0$ and $\beta>0$. Choose an arbitrary $\varepsilon \in(0, \min \{\alpha, \beta\} / 2)$. Then, there exist $t^{\prime} \in(0, t)$ and $s^{\prime} \in(0, s)$ such that

$\alpha-\varepsilon<M_{S}\left(\left(x_{n}\right)_{n},\left(y_{n}\right)_{n}, t^{\prime}\right)$ and $\beta-\varepsilon<M_{S}\left(\left(y_{n}\right)_{n},\left(z_{n}\right)_{n}, s^{\prime}\right)$.

Furthermore, there exists $n_{\varepsilon}$ such that for each $k \geq n_{\varepsilon}$,

$$
\begin{aligned}
& M_{S}\left(\left(x_{n}\right)_{n},\left(y_{n}\right)_{n}, t^{\prime}\right)-\varepsilon<M\left(x_{k}, y_{k}, t^{\prime}\right), \text { and } \\
& M_{S}\left(\left(y_{n}\right)_{n},\left(z_{n}\right)_{n}, s^{\prime}\right)-\varepsilon<M\left(y_{k}, z_{k}, s^{\prime}\right) .
\end{aligned}
$$

Then

$$
\begin{aligned}
(\alpha-2 \varepsilon) *(\beta-2 \varepsilon) & \leq\left(M_{S}\left(\left(x_{n}\right)_{n},\left(y_{n}\right)_{n}, t^{\prime}\right)-\varepsilon\right) *\left(M_{S}\left(\left(y_{n}\right)_{n},\left(z_{n}\right)_{n}, s^{\prime}\right)-\varepsilon\right) \\
& \leq M\left(x_{k}, y_{k}, t^{\prime}\right) * M\left(y_{k}, z_{k}, s^{\prime}\right) \\
& \leq M\left(x_{k}, z_{k}, t^{\prime}+s^{\prime}\right) .
\end{aligned}
$$

for all $k \geq n_{\varepsilon}$. 
Therefore

$$
\begin{aligned}
(\alpha-2 \varepsilon) *(\beta-2 \varepsilon) & \leq \inf _{k \geq n_{\varepsilon}} M\left(x_{k}, z_{k}, t^{\prime}+s^{\prime}\right) \leq \underline{\lim } M\left(x_{n}, z_{n}, t^{\prime}+s^{\prime}\right) \\
& \leq \sup _{0<r<t+s} \underline{\lim } M\left(x_{n}, z_{n}, t^{\prime}+s^{\prime}\right)=\gamma .
\end{aligned}
$$

By continuity of $*$, it follows that $\alpha * \beta \leq \gamma$. So condition (KM3) is satisfied.

Finally, fix $\left(x_{n}\right)_{n},\left(y_{n}\right)_{n} \in S$ and $t>0$, and let $\left(t_{j}\right)_{j}$ be a strictly increasing sequence of positive real numbers such that $\lim _{j} t_{j}=t$. Since $M\left(x_{k}, y_{k}, t_{j}\right) \leq$ $M\left(x_{k}, y_{k}, t\right)$, for all $k, j$, it follows that

$$
M_{S}\left(\left(x_{n}\right)_{n},\left(y_{n}\right)_{n}, t_{j}\right) \leq M_{S}\left(\left(x_{n}\right)_{n},\left(y_{n}\right), t\right),
$$

for all $j$.

Moreover, given $\varepsilon>0$, there is $s_{\varepsilon} \in(0, t)$ such that

$$
M_{S}\left(\left(x_{n}\right),\left(y_{n}\right), t\right)<\varepsilon+\sup _{n} \inf _{k \geq n} M\left(x_{k}, y_{k}, s_{\varepsilon}\right) .
$$

Let $j_{\varepsilon}$ such that $t_{j}>s_{\varepsilon}$ for all $j \geq j_{\varepsilon}$. From the preceding relation, we deduce that

$$
M_{S}\left(\left(x_{n}\right),\left(y_{n}\right), t\right) \leq \varepsilon+\sup _{0<s<t_{j}} \underline{\lim } M\left(x_{n}, y_{n}, s\right)
$$

for all $j \geq j_{\varepsilon}$. So

$$
M_{S}\left(\left(x_{n}\right)_{n},\left(y_{n}\right)_{n}, t_{j}\right) \leq M_{S}\left(\left(x_{n}\right),\left(y_{n}\right), t\right) \leq \varepsilon+M_{S}\left(\left(x_{n}\right)_{n},\left(y_{n}\right)_{n}, t_{j}\right),
$$

for all $j \geq j_{\varepsilon}$. We conclude that $M_{S}\left(\left(x_{n}\right)_{n},\left(y_{n}\right)_{n},{ }_{-}\right)$is left-continuous. Therefore, condition (KM4) is satisfied.

Now denote by $\widetilde{X}$ the quotient $S / \sim$, and by $\left[\left(x_{n}\right)_{n}\right]$ the class of the element $\left(x_{n}\right)_{n}$ of $S$.

Lemma 3. For each $\left(x_{n}\right)_{n},\left(y_{n}\right)_{n} \in S$ and each $\left(a_{n}\right)_{n} \in\left[\left(x_{n}\right)_{n}\right],\left(b_{n}\right)_{n} \in\left[\left(y_{n}\right)_{n}\right]$, one has

$$
M_{S}\left(\left(x_{n}\right)_{n},\left(y_{n}\right)_{n}, t\right)=M_{S}\left(\left(a_{n}\right)_{n},\left(b_{n}\right)_{n}, t\right),
$$


for all $t>0$.

Proof. Let $t>0$. Given $\varepsilon \in(0, t / 2)$ we obtain

$$
\begin{aligned}
& M_{S}\left(\left(x_{n}\right)_{n},\left(y_{n}\right)_{n}, t\right) \\
\geq & M_{S}\left(\left(x_{n}\right)_{n},\left(a_{n}\right)_{n}, \varepsilon\right) * M_{S}\left(\left(a_{n}\right)_{n},\left(b_{n}\right)_{n}, t-2 \varepsilon\right) * M_{S}\left(\left(b_{n}\right)_{n},\left(y_{n}\right)_{n}, \varepsilon\right) \\
= & M_{S}\left(\left(a_{n}\right)_{n},\left(b_{n}\right)_{n}, t-2 \varepsilon\right) .
\end{aligned}
$$

Since $M_{S}\left(\left(a_{n}\right)_{n},\left(b_{n}\right)_{n},{ }_{-}\right)$is left continuous, we deduce that

$$
\lim _{\varepsilon \rightarrow 0} M_{S}\left(\left(a_{n}\right)_{n},\left(b_{n}\right)_{n}, t-2 \varepsilon\right)=M_{S}\left(\left(a_{n}\right)_{n},\left(b_{n}\right)_{n}, t\right) .
$$

Thus $M_{S}\left(\left(x_{n}\right)_{n},\left(y_{n}\right)_{n}, t\right) \geq M_{S}\left(\left(a_{n}\right)_{n},\left(b_{n}\right)_{n}, t\right)$.

The same argument shows that $M_{S}\left(\left(a_{n}\right)_{n},\left(b_{n}\right)_{n}, t\right) \geq M_{S}\left(\left(x_{n}\right)_{n},\left(y_{n}\right)_{n}, t\right)$

Lemma $4([9])$. Let $(X, M, *)$ be a fuzzy quasi-metric space and $(Y, N, \star)$ a

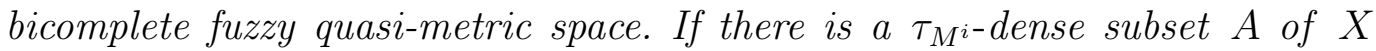
and an isometry $f:(A, M, *) \rightarrow(Y, N, \star)$, then there exists a unique isometry $F:(X, M, *) \rightarrow(Y, N, \star)$ such that $\left.F\right|_{A}=f$.

Now, for each $\left[\left(x_{n}\right)_{n}\right],\left[\left(y_{n}\right)_{n}\right] \in \widetilde{X}$, define

$$
\widetilde{M}\left(\left[\left(x_{n}\right)_{n}\right],\left[\left(y_{n}\right)_{n}\right], 0\right)=0,
$$

and

$$
\widetilde{M}\left(\left[\left(x_{n}\right)_{n}\right],\left[\left(y_{n}\right)_{n}\right], t\right)=M_{S}\left(x_{n}, y_{n}, t\right),
$$

for $t>0$.

Then $\widetilde{M}$ is a function from $\widetilde{X} \times \widetilde{X} \times[0, \infty)$ to $[0,1]$ (indeed, it is well-defined by Lemma 3$)$.

We also define $i: X \rightarrow \widetilde{X}$ such that, for each $x \in X, i(x)$ is the class of the constant sequence $x, x, \ldots$

From the above constructions we obtain:

Theorem 1. Let $(X, M, *)$ be a fuzzy quasi-metric space. Then: 
(a) $(\widetilde{M}, *)$ is a fuzzy quasi-metric on $\widetilde{X}$.

(b) $i(X)$ is dense in $\left(\widetilde{X}, \widetilde{M}^{i}, *\right)$.

(c) $(X, M, *)$ is isometric to $(i(X), \widetilde{M}, *)$.

(d) $(\widetilde{M}, *)$ is bicomplete.

(e) If $(Y, N, *)$ is a bicomplete fuzzy quasi-metric space such that $(X, M, *)$ is isometric to a $\tau_{N^{i}}$-dense subspace of $Y$, then $(Y, N, *)$ and $(\widetilde{X}, \widetilde{M}, *)$ are isometric.

Proof. (a) $(\widetilde{M}, *)$ satisfies conditions (KM1), (KM3) and (KM4) of Definition 1 as an immediate consequence of Lemma 2.

Now let $\left(x_{n}\right)_{n},\left(y_{n}\right)_{n} \in S$ such that $\widetilde{M}\left(\left[\left(x_{n}\right)_{n}\right],\left[\left(y_{n}\right)_{n}\right], t\right)=1$ for all $t>0$. If $\left(z_{n}\right)_{n} \in\left[\left(y_{n}\right)_{n}\right]$, it follows from Lemma 3 that $M_{S}\left(\left(z_{n}\right)_{n},\left(y_{n}\right)_{n}, t\right)=1$ for all $t>0$, i.e., $\left(z_{n}\right)_{n} \in\left[\left(y_{n}\right)_{n}\right]$. The same argument shows that $\left(z_{n}\right)_{n} \in\left[\left(x_{n}\right)_{n}\right]$ whenever $\left(z_{n}\right)_{n} \in\left[\left(y_{n}\right)_{n}\right]$. We conclude that $\widetilde{M}\left(\left[\left(x_{n}\right)_{n}\right],\left[\left(y_{n}\right)_{n}\right], t\right)=1$ for all $t>0$, if and only if $\left[\left(x_{n}\right)_{n}\right]=\left[\left(y_{n}\right)_{n}\right]$. Consequently $(\widetilde{M}, *)$ is a fuzzy quasimetric on $\widetilde{X}$.

(b) Let $\left(x_{n}\right)_{n} \in S, \varepsilon \in(0,1)$ and $t>0$. Choose an $s_{\varepsilon} \in(0, t)$. Since $\left(x_{n}\right)_{n}$ is a Cauchy sequence in $\left(X, M^{i}, *\right)$ there is $n_{\varepsilon} \in \mathbb{N}$ such that $M^{i}\left(x_{k}, x_{n_{\varepsilon}}, s_{\varepsilon}\right)>$ $1-\varepsilon / 2$ for all $k \geq n_{\varepsilon}$. Thus

$$
\begin{aligned}
\widetilde{M}\left(\left[\left(x_{n}\right)_{n}\right], i\left(x_{n_{\varepsilon}}\right), t\right) & =\sup _{0<s<t} \sup _{n} \inf _{k \geq n} M\left(x_{k}, x_{n_{\varepsilon}}, s\right) \\
& \geq \sup _{n} \inf _{k \geq n} M\left(x_{k}, x_{n_{\varepsilon}}, s_{\varepsilon}\right) \\
& \geq \inf _{k \geq n_{\varepsilon}} M\left(x_{k}, x_{n_{\varepsilon}}, s_{\varepsilon}\right) \\
& \geq \inf _{k \geq n_{\varepsilon}} M^{i}\left(x_{k}, x_{n_{\varepsilon}}, s_{\varepsilon}\right) \\
& \geq 1-\varepsilon / 2>1-\varepsilon .
\end{aligned}
$$

Similarly, we deduce that

$$
\widetilde{M}^{-1}\left(\left[\left(x_{n}\right)_{n}\right], i\left(x_{n_{\varepsilon}}\right), t\right)>1-\varepsilon .
$$

We have shown that $i(X)$ is dense in $\left(\widetilde{X}, \widetilde{M}^{i}, *\right)$.

(c) This is almost obvious because for each $x, y \in X$ and $t>0$ we have

$$
\widetilde{M}(i(x), i(y), t)=\sup _{0<s<t} M(x, y, s)=M(x, y, t) .
$$


(d) Let $\left(\widetilde{x}_{n}\right)_{n}$ be a Cauchy sequence in $\left(\widetilde{X}, \widetilde{M}^{i}, *\right)$. Then, there is an increasing sequence $\left(n_{k}\right)_{k}$ in $\mathbb{N}$ such that

$$
\widetilde{M}^{i}\left(\widetilde{x}_{n}, \widetilde{x}_{m}, 2^{-k}\right)>1-2^{-k}
$$

for all $n, m \geq n_{k}$.

Since $i(X)$ is dense in $\left(\widetilde{X}, \widetilde{M}^{i}, *\right)$, for each $k \in \mathbb{N}$ there is $y_{k} \in X$ such that

$$
\widetilde{M}^{i}\left(\widetilde{x}_{n_{k}}, i\left(y_{k}\right), 2^{-k}\right)>1-2^{-k}
$$

for all $k \in \mathbb{N}$.

We show that $\left(y_{k}\right)_{k}$ is a Cauchy sequence in $\left(X, M^{i}, *\right)$. To this end, choose $\varepsilon \in(0,1)$ and $t>0$. Take $j \in \mathbb{N}$ such that $2^{-j}<t / 3$ and

$$
\left(1-2^{-j}\right) *\left(1-2^{-j}\right) *\left(1-2^{-j}\right)>1-\varepsilon .
$$

Then, for each $k, m \geq j$, we have

$$
\begin{aligned}
M\left(y_{k}, y_{m}, t\right) & =\widetilde{M}\left(i\left(y_{k}\right), i\left(y_{m}\right), t\right) \geq \widetilde{M}\left(i\left(y_{k}\right), i\left(y_{m}\right), 3 \cdot 2^{-j}\right) \\
& \geq \widetilde{M}\left(i\left(y_{k}\right), \widetilde{x}_{n_{k}}, 2^{-j}\right) * \widetilde{M}\left(\widetilde{x}_{n_{k}}, \widetilde{x}_{n_{m}}, 2^{-j}\right) * \widetilde{M}\left(\widetilde{x}_{n_{m}}, i\left(y_{m}\right), 2^{-j}\right) \\
& \geq \widetilde{M}\left(i\left(y_{k}\right), \widetilde{x}_{n_{k}}, 2^{-k}\right) * \widetilde{M}\left(\widetilde{x}_{n_{k}}, \widetilde{x}_{n_{m}}, 2^{-(k \wedge m)}\right) * \widetilde{M}\left(\widetilde{x}_{n_{m}}, i\left(y_{m}\right), 2^{-m}\right) \\
& \geq\left(1-2^{-k}\right) *\left(1-2^{-(\min \{k, m\})}\right) *\left(1-2^{-m}\right) \\
& \geq\left(1-2^{-j}\right) *\left(1-2^{-j}\right) *\left(1-2^{-j}\right)>1-\varepsilon,
\end{aligned}
$$

and consequently $\left(y_{k}\right)_{k}$ is a Cauchy sequence in $\left(X, M^{i}, *\right)$. Therefore $\widetilde{y} \in \widetilde{X}$, where $\widetilde{y}:=\left[\left(y_{k}\right)_{k}\right]$.

Finally, we prove that the sequence $\left(\widetilde{x}_{n}\right)_{n}$ converges to $\widetilde{y}$ in $\left(\widetilde{X}, \widetilde{M}^{i}, *\right)$. Indeed, as in part (c) above, choose $\varepsilon \in(0,1)$ and $t>0$. Take $j \in \mathbb{N}$ such that $2^{-j}<t / 3$, and

$$
\left(1-2^{-j}\right) *\left(1-2^{-j}\right) *\left(1-2^{-j}\right)>1-\varepsilon .
$$

Since $\left(y_{k}\right)_{k}$ is a Cauchy sequence in $\left(\widetilde{X}, \widetilde{M}^{i}, *\right)$, the proof of part (b) shows that there is $k \geq j$ such that

$$
\widetilde{M}^{i}\left(\widetilde{y}, i\left(y_{k}\right), 2^{-j}\right)>1-2^{-j}
$$

Then, for $n \geq n_{k}$ we obtain 


$$
\begin{aligned}
\widetilde{M}^{i}\left(\widetilde{y}, \widetilde{x}_{n}, t\right) & \geq \widetilde{M}^{i}\left(\widetilde{y}, i\left(y_{k}\right), 2^{-j}\right) * \widetilde{M}^{i}\left(i\left(y_{k}\right), \widetilde{x}_{n_{k}}, 2^{-j}\right) * \widetilde{M}^{i}\left(\widetilde{x}_{n_{k}}, \widetilde{x}_{n}, 2^{-j}\right) \\
& \geq\left(1-2^{-j}\right) * \widetilde{M}^{i}\left(i\left(y_{k}\right), \widetilde{x}_{n_{k}}, 2^{-k}\right) * \widetilde{M}^{i}\left(\widetilde{x}_{n_{k}}, \widetilde{x}_{n}, 2^{-k}\right) \\
& \geq\left(1-2^{-j}\right) *\left(1-2^{-k}\right) *\left(1-2^{-k}\right) \\
& \geq\left(1-2^{-j}\right) *\left(1-2^{-j}\right) *\left(1-2^{-j}\right)>1-\varepsilon .
\end{aligned}
$$

We conclude that $(\widetilde{X}, \widetilde{M}, *)$ is bicomplete.

(e) This follows directly from Lemma 4 and standard arguments.

Remark 2. The preceding theorem implies that every fuzzy quasi-metric space $(X, M, *)$ has a bicompletion which is unique up to isometry. We refer to $(\widetilde{X}, \widetilde{M}, *)$ as the bicompletion of $(X, M, *)$.

Remark 3. Note that if $(X, M, *)$ is a fuzzy quasi-metric space, then $\widetilde{M}^{-1}=$ $\widetilde{M^{-1}}$ on $\widetilde{X}$. On the other hand, if $(X, M, *)$ is a fuzzy metric space, then $(\widetilde{M}, *)$ is a fuzzy metric on $\widetilde{X}$, and thus the complete fuzzy metric space $(\widetilde{X}, \widetilde{M}, *)$ is the completion of $(X, M, *)$.

From Theorem 1 and Remark 3 we deduce the following result which essentially shows that the for each fuzzy quasi-metric space, the completion of its induced fuzzy metric space coincides with the fuzzy metric space induced by its bicompletion.

Proposition 1. Let $(X, M, *)$ be a fuzzy quasi-metric space. Then the (complete) fuzzy metrics $\left(\widetilde{M^{i}}, *\right)$ and $\left(\widetilde{M}^{i}, *\right)$ coincide on $\widetilde{X}$.

Proof. By Theorem 1, (b) and (d), $\left(\widetilde{X}, \widetilde{M}^{i}, *\right)$ is a complete fuzzy metric space that contains $i(X)$ has a dense subspace. On the other hand, it follows from Remark 3 and the construction of $\widetilde{X}$ that $\left(\widetilde{X}, \widetilde{M^{i}}, *\right)$ is the completion of $\left(X, M^{i}, *\right)$. Therefore $\left(\widetilde{X}, \widetilde{M}^{i}, *\right)$ and $\left(\widetilde{X}, \widetilde{M^{i}}, *\right)$ coincide by the uniqueness of the completion, i.e., the (complete) fuzzy metrics $\left(\widetilde{M^{i}}, *\right)$ and $\left(\widetilde{M}^{i}, *\right)$ coincide on $\widetilde{X}$

In the next we apply our constructions to obtain the relationship between the bicompletion of the quasi-metric spaces and of the fuzzy quasi-metric spaces of Examples 1 and 2, respectively. In order to help to the reader we recall the construction of the bicompletion of a quasi-metric space (see $[2,19]$ or p. 163 of [13]). 
Let $(X, d)$ be a quasi-metric space. Denote by $Y$ the set of all Cauchy sequences in the metric space $\left(X, d^{s}\right)$. For each $\left(x_{n}\right)_{n},\left(y_{n}\right)_{n} \in Y$ put $\left(x_{n}\right)_{n} \sim\left(y_{n}\right)_{n}$ if and only if $\lim _{n} d^{s}\left(x_{n}, y_{n}\right)=0$. Then $\sim$ is an equivalence relation on $Y$. Denote by $X^{B}$ the quotient $Y / \sim$. For each $\left[\left(x_{n}\right)_{n}\right],\left[\left(y_{n}\right)_{n}\right] \in X^{B}$, let $d^{B}\left(\left[\left(x_{n}\right)_{n}\right],\left[\left(y_{n}\right)_{n}\right]\right)=\lim _{n} d\left(x_{n}, y_{n}\right)$. Then $\left(X^{B}, d^{B}\right)$ is a bicomplete quasimetric space such that $(X, d)$ is isometric to a dense subspace of the metric space $\left(X^{B},\left(d^{B}\right)^{s}\right)$. The space $\left(X^{B}, d^{B}\right)$ is said to be the bicompletion of $(X, d)$. Furthermore, the bicompletion coincides with the standard completion when $(X, d)$ is a metric space.

Example 4. Let $(X, d)$ be a quasi-metric space and let $\left(X, M_{d, 01}, *\right)$ be the fuzzy quasi-metric space of Example 1. It is almost obvious that a sequence in $X$ is a Cauchy sequence in $\left(X, d^{s}\right)$ if and only if it is a Cauchy sequence in $\left(X,\left(M_{d, 01}\right)^{i}, *\right)$, and thus it easily follows that $\widetilde{X}=X^{B}$. For each $\left[\left(x_{n}\right)_{n}\right],\left[\left(y_{n}\right)_{n}\right] \in \widetilde{X}$ and $t>0$ we have

$$
d^{B}\left(\left[\left(x_{n}\right)_{n}\right],\left[\left(y_{n}\right)_{n}\right]\right)<t \Leftrightarrow \widetilde{M_{d, 01}}\left(\left[\left(x_{n}\right)_{n}\right],\left[\left(y_{n}\right)_{n}\right]\right)=1,
$$

and hence $\widetilde{M_{d, 01}}=M_{d^{B}, 01}$ on $\widetilde{X}$.

Example 5. Let $(X, M, *)$ be a fuzzy quasi-metric space such that $*_{L} \leq *$. It is clear that a sequence in $X$ is Cauchy in $\left(X,\left(d_{M}\right)^{s}\right)$ if and only if it is Cauchy in $\left(X, M^{i}, *\right)$. We show that $\left(d_{M}\right)^{B}=d_{\widetilde{M}}$ on $X^{B}$. Indeed, first note that for $\left[\left(x_{n}\right)_{n}\right],\left[\left(y_{n}\right)_{n}\right] \in X^{B}$ and $t>0$, one has $t \leq 1-\widetilde{M}\left(\left[\left(x_{n}\right)_{n}\right],\left[\left(y_{n}\right)_{n}\right], t\right)$ whenever $t \leq 1-M\left(x_{n}, y_{n}, t\right)$ eventually, and consequently $\left(d_{M}\right)^{B} \leq d_{\widetilde{M}}$. On the other hand, given $t>0$ with $t \leq 1-\widetilde{M}\left(\left[\left(x_{n}\right)_{n}\right],\left[\left(y_{n}\right)_{n}\right], t\right)$, for each $\varepsilon \in(0, t / 2)$ and $s \in(0, t)$ with $t<s+\varepsilon$ we can find an $n_{s}$ such that $M\left(x_{n_{s}} y_{n_{s}}, s\right)<1-t+\varepsilon$, so that $s<\varepsilon+1-M\left(x_{n_{s}}, y_{n_{s}}, s\right) \leq \varepsilon+1-M\left(x_{n_{1}}, y_{n_{1}}, s-\varepsilon\right)$, and thus $t<s+\varepsilon<$ $2 \varepsilon+\sup \left\{r \geq 0: r \leq 1-M\left(x_{n}, y_{n}, r\right)\right\}$. Hence $t \leq\left(d_{M}\right)^{B}\left(\left[\left(x_{n}\right)_{n}\right],\left[\left(y_{n}\right)_{n}\right]\right)$, so that $d_{\widetilde{M}} \leq\left(d_{M}\right)^{B}$.

We conclude the paper with some observations on the (bi)completion of GVfuzzy (quasi-)metric spaces, for which the situation is quite different to the corresponding one for KM-fuzzy (quasi-)metric spaces.

Remark 4. In Example 2 of [10] it was shown that if $(X, d)$ is a quasimetric space, then the bicompletion $\left(\widetilde{X}, \widetilde{M_{d}}, *\right)$ of the standard GV-fuzzy quasi-metric space $\left(X, M_{d}, *\right)$ is (isometric to) the $\mathrm{GV}$-fuzzy quasi-metric space $\left(X^{B}, M_{d^{B}}, *\right)$, i.e., the bicompletion of the standard GV-fuzzy quasi-metric space $\left(X, M_{d}, *\right)$ is the standard GV-fuzzy quasi-metric space of the bicom- 
pletion of $(X, d)$. On the other hand, it was given in [7] an example of a GVfuzzy metric space whose completion is not a GV-fuzzy metric space, and in [8], it was obtained a characterization of those GV-fuzzy metric spaces whose completion is a GV-fuzzy metric space. (This characterization was extended to the fuzzy quasi-metric framework in [10].)

\section{Acknowledgement}

The authors thank to one of reviewers for suggesting the question discussed in Proposition 1.

\section{References}

[1] Y. J. Cho, M. Grabiec and V. Radu, On non Symmetric Topological and Probabilistic Structures, Nova Science Publisher, Inc. New York, 2006.

[2] A. Di Concilio, Spazi quasimetrici e topologie ad essi associate, Rend. Accad. Sci. Fis. Mat. Napoli 38 (1971), 113-130.

[3] P. Fletcher, W.F. Lindgren, Quasi-Uniform Spaces, Marcel Dekker, New York, 1982.

[4] A. George, P. Veeramani, On some results in fuzzy metric spaces, Fuzzy Sets and Systems 64 (1994), 395-399.

[5] A. George, P. Veeramani, On some results of analysis of fuzzy metric spaces, Fuzzy Sets and Systems 90 (1997), 365-368.

[6] B.V. Gnedenko, A.N. Kolmogorov, Limit Distributions for Sums of Independent Random Variables, Cambridge, Addison-Wesley, 1954.

[7] V. Gregori, S. Romaguera, On completion of fuzzy metric spaces, Fuzzy Sets and Systems 130 (2002), 399-404.

[8] V. Gregori, S. Romaguera, Characterizing completable fuzzy metric spaces, Fuzzy Sets and Systems 144 (2004), 411-420.

[9] V. Gregori, S. Romaguera, Fuzzy quasi-metric spaces, Appl. Gen. Topology 5 (2004), 129-136.

[10] V. Gregori, S. Romaguera, A. Sapena, A characterization of bicompletable fuzzy quasi-metric spaces, Fuzzy Sets and Systems 152 (2005), 395-402.

[11] I. Kramosil, J. Michalek, Fuzzy metrics and statistical metric spaces, Kybernetika 11 (1975), 326-334.

[12] H.P.A. Künzi, Nonsymmetric distances and their associated topologies: About the origins of basic ideas in the area of asymmetric topology, in: Handbook 
of the History of General Topology, C.E. Aull, R. Lowen (Eds.), vol. 3, pp. 853-968, Kluwer, Dordrecht, 2001.

[13] H.P.A. Künzi, M.P. Schellekens, On the Yoneda completion of a quasi-metric spaces, Theoret. Comp. Sci. 278 (2002), 159-194.

[14] M. Loève, Probability Theory, New York, Van Nostrand, 1963.

[15] J. Rodríguez-López, S. Romaguera, J.M. Sánchez-Álvarez, The Hausdorff fuzzy quasi-metric, Fuzzy Sets and Systems 161 (2010), 1078-1096.

[16] S. Romaguera, A. Sapena, P. Tirado, The Banach fixed point theorem in fuzzy quasi-metric spaces with application to the domain of words, Topology Appl. 154 (2007), 2196-2203.

[17] S. Romaguera, P. Tirado, Contraction maps on ifqm-spaces with application to recurrence equations of Quicksort, Elect. Notes Theoret. Comput. Sci. 225 (2009), 269-279.

[18] R. Saadati, S.M. Vaezpour and Y.J. Cho, Quicksort algorithm: Application of a fixed point theorem in intuitionistic fuzzy quasi-metric spaces at a domain of words, J. Comput. Appl. Math. 228 (2009), 219-225.

[19] S. Salbany, Bitopological Spaces, Completions and Compactifications, Math. Monographs, no 1, Dept. Math. Univ. Cape Town, 1974.

[20] B. Schweizer, A. Sklar, Statistical metric spaces, Pacific J. Math. 10 (1960), 314-334.

[21] H. Sherwood, On the completion of probabilistic metric spaces, Z. Wahrsch. verw. Geb. 6 (1966), 62-64. 